\title{
Carbon Emission Trends of Agricultural Product Proceeding Industry in China
}

\author{
Zhongyin Sheng ${ }^{1}$, Quansheng Gao ${ }^{1 *}$ \\ ${ }^{1}$ Department of Mathematics and Computer Science, \\ Wuhan Polytechnic University, \\ Wuhan, 430023, China
}

\author{
Dali Xiong ${ }^{2}$ \\ ${ }^{2}$ Department of Foreign Languages, \\ China University of Geosciences, \\ Wuhan, 430074, China \\ *corresponding author, e-mail: 71703441@qq.com
}

\begin{abstract}
In this paper we investigate the carbon dioxide emission trends of China's agricultural product proceeding industry from 1995 to 2011 with index decomposition analysis. The results show that carbon emission of the agricultural product proceeding sub-sectors presents an obviously upward trend with time series and there is a slowdown in the increase of carbon emission in recent years. The top three industries are textile industry, paper making and paper products industry and food manufacturing all the time. As to the influencing factors behind the stagnancy of carbon emission, the GDP per capita, energy structure and total population have a significant impact on carbon emission.
\end{abstract}

Keywords-agricultural product proceeding industry; carbon emission; index decomposition analysis

\section{INTRODUCTION}

In recent years, with rapid development of China's agricultural product proceeding industry, which has become one of the pillar industries of the national economy, the consequent increase in energy consumption and carbon emission and other issues are also coming, but have not yet caused widespread concern. At present, almost investigates about carbon emission are discussed around heavy industry ,but it's not often seen in agricultural product proceeding industry .In this paper, we intends to use the related theory of carbon emission, from temporal trends, structure state, affecting factors and the overall trends these four aspects to explore $\mathrm{CO} 2$ emission trends of China's agricultural product proceeding industry, clarify the basic law of the agro-industrial carbon emission, enrich and broaden the content of carbon theory system.

Since the 1980s, index decomposition method has become the main tool of international energy analysis and energy policy formulation. The common methods are Adaptive Weight Decomposition (AWD), Laspeyres index, the Simple Average Decomposition (SAD), Logarithmic Mean Disivia Index (LMDI) and so on. While the index decomposition analysis was used in carbon emission research for decades, many domestic and foreign scholars strived to focus on carbon emissionand climate change, carbon emission measures and influencing factors and so on, finally they obtained some valuable achievements. Wang (2005) decomposed the affecting factors of carbon emission into population scale,
GDP per capita, energy efficiency, fossil energy ratio and carbon intensity of fossil energy. Then researched the carbon emission in China during years 1957-2000 basing on the LMDI method and obtained the result: energy intensity is the most important factor in decreasing the carbon emission, then in the energy structure, while the growing economy will promote the carbon emission [1].Stan(2007) explored the impact mechanism of carbon emission, from economic structure, growth and energy efficiency, as well as making specific policy and recommendation[2]. Xu Guoquan (2006) studied the carbon emission in China from 1995 to 2004 using the SAD method, and drew a conclusion: carbon emission per capita increases exponentially with economic development, reduces presenting inverted U-shape with the energy structure and energy efficiency[3].Xu Guangyue and Song Deyong(2010) studied the problem of CO2 emission from 1980 to 2007 of our country and analyzed the long-term co-integration relationship of carbon emission, economic growth and export trade dynamically through index decomposition analysis [4]. These studies provide theoretical support to the further analysis of carbon emissio, as well as offer the satisfactory basis to taking measures to energy conservation and emission reduction.

\section{THE METHOD AND ASSUMPTIONS}

\section{A. Research Method}

The index decomposition analysis method using in the carbon emission research of agricultural products processing industry works as follows :firstly determine the weight of different potential elements according to the affecting degree of carbon emission making by these elements, then the product of these affecting factors is the carbon dioxide emission .The current rapid development of China's economy determines the increasing energy demand, the coal-focused energy structure determines the status of the carbon gas emission. What impacts the CO2 emission are mainly following four factors:

i) Total CO2 emission .With China's rapid economic development, energy consumption has increased rapidly in recent years. In 2011, our CO2 emission accounts for more than $25 \%$ of the world's total carbon emission.

ii) Carbon intensity of energy , which is the $\mathrm{CO} 2$ emission per unit of energy consumption. The datum in 2008 reached 2.87 tons of carbon dioxide according to 
standard oil per ton .It is 1.26 times of the world average [5].

iii) Carbon intensity of GDP, which means CO2 emission produced by per unit of GDP. According to statistics, GDP is calculated at comparable price in 2000, the value of this factor is 0.59 dollar $/ \mathrm{kg}$ of our country in 2008 compare to 0.46 dollar $/ \mathrm{kg}$ of the world average level.

iv) Energy consumption per unit of GDP (energy intensity).It refers to that in a period of time ,the amount of energy consumption in a country or region produced by one unit of domestic or regional production gross .According to Energy Statistical Yearbook, in 2011, China's energy consumption per unit of GDP was 0.79 tons of standard coal /ten thousands (GDP).This datum is twice of the world average (GDP is calculated at comparable price in 2010).

\section{B. Research hypothesis}

i) According to the classification standards in China, agricultural product proceeding industry mainly includes 12 sub-industries :food processing industry (sector 1), food manufacturing (sector 2), beverage manufacturing (sector 3),tobacco industry (sector 4), textile industry (sector 5),clothing and other fiber products manufacturing (sector 6),leather ,fur ,eiderdown and related products (sector 7), wood processing and grass bamboo rattan palm products (sector 8), furniture manufacturing (sector 9), paper making and paper products industry (sector 10), printing industry and copy of recording medium (sector 11 ), rubber products industry (sector 12 ).

ii) Assuming fuel for the processing of agricultural products is complete combustion, and the carbonaceous product during burning has only carbon dioxide. According to the Kyoto Protocol, there are six controlled greenhouse gases, of which $\mathrm{CO} 2$ is the most and contributes the largst to the global warming ,thus in this paper, we only consider carbon dioxide of all the carbon emission forms.

iii) Assuming standard coal carbon emission factor is 2.8.We get the factor of standard coal indirectly via calculating the $\mathrm{CO} 2$ emission of crude oil.

iv) Assuming the industry's energy consumption per unit of GDP remains unchanged, namely an energy same distribution across all sectors.

\section{CARBON EMISSION TRENDS OF AGRICULTURAL PRODUCT PROCEEDING INDUSTRY IN CHINA}

\section{A. Temporal trends of carbon emission of the} agricultural product proceeding sub-sectors

Total carbon dioxide emission can be estimated by the following formula:

$$
A=\sum_{i=1}^{12} A_{i}=\sum_{i=1}^{12}\left(D_{i} \times \alpha\right)
$$

Where, $A$ is the total carbon dioxide emission of agricultural product proceeding industry, $A_{i}$ is the carbon dioxide emission of sub-sectors $(i=1,2 \cdots 12) . D_{i}$ stands for energy consumption of standard coal of industrial divisions. $\alpha$ is standard coal carbon emission coefficient, $\alpha=2.8$.

We can obtain the total $\mathrm{CO} 2$ emission of agricultural product proceeding industry during years 1995-2011 with formula (1), as shown in Table I. As seen from the table, the $\mathrm{CO} 2$ emission of sub-sectors presents increasing trend year by year and reaches the greatest between 2000 and 2005, then slows down. The change trend is the same with the energy consumption of standard coal during years 1995-2011. It is the rapid development of China's industrialization determines the increase of energy consumption.

TABLE I. TOTAL $\mathrm{CO}_{2}$ EMISSION OF SUB-SECTORS FROM 1995-2011(UNIT:104 TONS)

\begin{tabular}{cccccccccc}
\hline & 1995 & 2000 & 2005 & 2006 & 2007 & 2008 & 2009 & 2010 & 2011 \\
\hline Sector1 & 6310.9 & 5492.4 & 7064.1 & 7551.3 & 8119.7 & 8738.5 & 8942.0 & 9135.4 & 9295.7 \\
Sector 2 & 3862.5 & 3373.4 & 4102.7 & 4445.6 & 4633.3 & 4939.7 & 4999.3 & 5101.8 & 5213.5 \\
Sector 3 & 3199.3 & 2559.3 & 3175.7 & 3459.4 & 3514.4 & 3715.6 & 3809.8 & 3896.4 & 4032.6 \\
Sector 4 & 714.1 & 970.5 & 823.1 & 816.8 & 795.7 & 743.3 & 747.5 & 760.1 & 755.7 \\
Sector 5 & 11297.4 & 9644.5 & 16897.4 & 19545.2 & 20889.3 & 20467.9 & 19998.9 & 19578.4 & 19288.5 \\
Sector 6 & 1051.7 & 1113.5 & 1846.5 & 2113.6 & 2274.2 & 2319.5 & 2279.4 & 2258.6 & 2215.3 \\
Sector 7 & 926.4 & 649.7 & 1034.5 & 1164.3 & 1238.3 & 1242.7 & 1229.1 & 1213.5 & 1209.5 \\
Sector 8 & 1215.6 & 1153.5 & 2416.3 & 2694.5 & 2857.4 & 3139.4 & 3356.7 & 3512.5 & 3697.4 \\
Sector 9 & 337.4 & 322.8 & 426.2 & 476.4 & 486.5 & 579.3 & 587.4 & 602.5 & 619.5 \\
Sector10 & 6839.5 & 7259.4 & 11438.4 & 12132.5 & 11657.4 & 12793.2 & 13124.6 & 13521.4 & 13932.5 \\
Sector 11 & 649.4 & 653.4 & 897.8 & 969.4 & 1045.5 & 1118.4 & 1142.4 & 1183.4 & 1203.5 \\
Sector 12 & 2059.5 & 2198.4 & 3637.3 & 3989.4 & 4175.3 & 4273.7 & 4301.3 & 4335.2 & 4352.7 \\
Total & 38463.7 & 35390.8 & 53760.0 & 59358.4 & 61687.0 & 64071.2 & 64518.4 & 65099.2 & 65816.4 \\
\hline
\end{tabular}

B. Structure state of carbon emission of the agricultural product proceeding sub-sectors

Total carbon dioxide emission also can be expressed as follows:

$$
A=\sum_{i=1}^{12} A_{i}=\sum_{i=1}^{12}\left(E \times \frac{E_{i}}{E} \times \frac{A_{i}}{E_{i}}\right)=\sum_{i=1}^{12}\left(E \times e_{i} \times S_{i}\right)
$$

Where, $E$ is total GDP of agricultural processing industry, $E_{i}$ is total GDP of sector $i$,carbon intensity of 
GDP of sector $i \quad S_{i}=A_{i} / E_{i}$,industrial structure $e_{i}=E_{i} / E$.

From the formula(2), the industrial structure of agricultural processing industry is an important factor of the total carbon dioxide emission. By calculating the GDP and total GDP data of sub-sectors, we can draw the conclusion that the industrial structure of agricultural processing industry in 2011 and overall industrial structure from 2005 to 2011 is consistent. Accordingly, carbon emission ratio of sub-sectors in 2011 and in 20052011 is broadly stable. The top three sectors contributed to the most carbon dioxide emission are always the textile industry, paper making and paper products industry and food manufacturing all the time. The carbon emission ratio of the above sectors respectively accounts for $29 \%$, $21 \%$ and $14 \%$ in 2011.

\section{Affecting factors of carbon emission of the agricultural product industry}

In this paper, Logarithmic mean disivia index (LMDI) method [6] which is put forward by Aug BW(1998),is adopted to decomposition and analyze the influencing factors of $\mathrm{CO} 2$ emission of agricultural processing industry .LMDI is a complete and non-residual decomposition analysis. It is more suitable for the condition of less factors and has more obvious advantage among all the index decomposition methods so far[7].The decomposition model of total CO2 emissions is given as:

$$
A=\sum_{j} \frac{C_{j}}{Q} \times \frac{F_{j}}{C_{j}} \times \frac{Q}{E} \times \frac{E}{N} \times N=\sum_{j} c_{j} \times M_{j} \times B \times G \times N
$$

Where $N$ is the total population, $Q$ is the total energy consumption of agricultural processing industry, $C_{j}$ is consumption of energy $j, F_{j}$ is $\mathrm{CO}_{2}$ emission of energy $j$. Carbon intensity of energy $j$ $M_{j}=F_{j} / C_{j}$,energy consumption per unit of GDP $B=Q / E$, GDP per capita $G=E / N$.Energy structure
$c_{j}=C_{j} / Q$, which means energy $j$ in the share of total energy consumption.

According to the model assumptions, energy consumption per unit of GDP remains unchanged, so symbol $B$ in the formula (3) can be regarded as a constant. The carbon emission intensity of coal, oil, gas, nuclear power, hydroelectricity and wind power are 0.7476, $0.5825,0.4435,0,0$ and 0 respectively . Therefore, $M_{j}$ is permanent each year. Then, there are three main affecting factors: energy structure, GDP per capita and total population.

Assuming the base period and the current period $\mathrm{CO}_{2}$ emissions were $A_{0}=\sum_{j} c_{j 0} M_{j 0} B_{0} G_{0} N_{0} \quad$ and $A_{t}=\sum_{j} c_{j t} M_{j t} B_{t} G_{t} N_{t}$.In addition mode, the change in carbon emissions between the base period and the current period is expressed as:

$$
\Delta A=A_{t}-A_{0}=\Delta A_{c}+\Delta A_{G}+\Delta A_{N}+\Delta A_{r s d}
$$

Where, $\Delta A_{c}, \Delta A_{G}, \Delta A_{N}$ are contribution of the energy structure, GDP per capita and population changes to $\mathrm{CO}_{2}$ emissions. In LMDI decomposition, residual $\Delta A_{r s d}=0$.Decomposition result of the above factors can be calculated as follows:

$$
\begin{aligned}
\Delta A_{c} & =\sum_{j} \frac{A_{j t}-A_{j 0}}{\ln A_{j t}-\ln A_{j 0}} \ln \frac{c_{j t}}{C_{j 0}} \\
\Delta A_{G} & =\sum_{j} \frac{A_{j t}-A_{j 0}}{\ln A_{j t}-\ln A_{j 0}} \ln \frac{G_{t}}{G_{0}} \\
\Delta A_{N} & =\sum_{j} \frac{A_{j t}-A_{j 0}}{\ln A_{j t}-\ln A_{j 0}} \ln \frac{N_{t}}{N_{0}}
\end{aligned}
$$

Base on the formula (5)-(7) and related data according to China Statistical Yearbook and China Energy Statistical Yearbook, we can get the decomposition value of these influencing factors, as shown in Table II:

TABLE II. THE DECOMPOSITION VALUE OF AFFECTING FACTORS (UNIT: 108 TONS)

\begin{tabular}{lllllllr}
\hline & 2000 & 2005 & 2006 & 2007 & 2008 & 2009 & 2010 \\
\hline Energy structure & 1.0263 & 1.5053 & 1.4646 & 1.4805 & 1.4736 & 1.4839 & 1.4322 \\
GDP per capita & 1.6988 & 2.6880 & 2.8305 & 2.9610 & 3.2036 & 3.2259 & 3.3050 \\
Population & 0.8140 & 1.1827 & 1.6408 & 1.7272 & 1.7299 & 1.7420 & 1.7728 \\
\hline
\end{tabular}

In China, coal in primary energy use accounted for

As can be seen from Table II, the energy structure, GDP per capita and population factors have a positive impact on the increase of carbon emission.GDP per capita, which corresponds to the economic development factor, contributes the most.The influence of population factor on carbon emission increases year by year. Huge population and the improvement of social comprehensive strength pose a challenge to the energy consumption. more than $65 \%$.The coal-focused energy structure, is not only one of the main reasons that China's CO2 emission has been so high, but also a key factor in the impact of carbon emission reduction .However, in recent years, with the widely use of low carbon energy and other clean energy, the energy structure in China is improved and its influence on carbon emission is reducing gradually. As shown in Table II. 


\section{Overall trends of carbon emission of the agricultural product proceeding}

From the point of industrial divisions, we discuss the relationship of $\mathrm{CO}_{2}$ emission $A$,energy consumption per unit of GDP $B$,carbon intensity of energy of the $i$ th sector $I_{i}$, GDP per capita $G$, total population $N$ and energy structure of the $i$ th sector $q_{i}$ :

$$
\begin{aligned}
A & =\sum_{i=1}^{12} A_{i}=\sum_{i=1}^{12}\left(\frac{A_{i}}{Q_{i}} \times \frac{Q_{i}}{Q} \times \frac{Q}{E} \times \frac{E}{N} \times N\right) \\
& =B \times G \times N \times \sum_{i=1}^{12}\left(I_{i} \times q_{i}\right)
\end{aligned}
$$

Where, $Q$ is the total energy consumption, $Q_{i}$ is the energy consumption of the sector $i$.

From the analysis above, carbon intensity of energy of the sectors, GDP per capita and total population are primary factors in influencing the $\mathrm{CO} 2$ emission .For the energy structure factor, total CO2 emission is not affected by it, but has close relationship with carbon emission of sectors. Under these combined effects, from 1995 to 2011,the increased state of total CO2 emission of agricultural product proceeding industry in a certain degree has relieved. As shown in Figure 1.

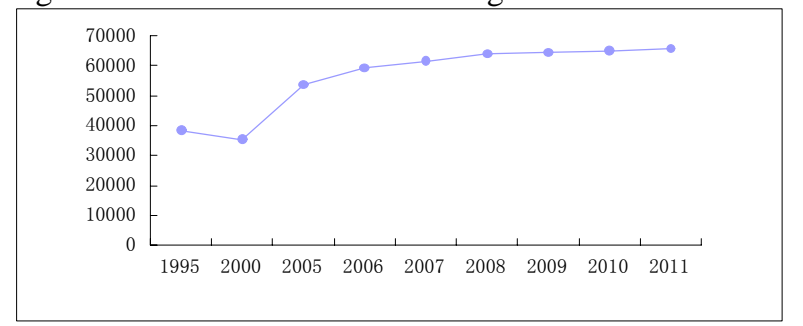

Figure 1. Overall trends of carbon emission(unit: $10^{4}$ tons)

\section{CONCLUSIONS AND SUGGESTIONS}

By analyzing the carbon emission of our agricultural product proceeding industry in 1995-2011 comprehensively based on index decomposition analysis , we get the conclusions as following : carbon emission of the agricultural product proceeding sub-sectors presents an obviously upward trend with time series, but the growing speed of carbon emission is gradually slowingdown in recent years. From the structure state of $\mathrm{CO} 2$ emission perspective, the industrial structure of subsectors remained the same from 2005-2011, and the emission ratios are also roughly stable. The top three industries are textile industry, paper making and paper products industry and food manufacturing all the time. About influencing factors of carbon emission, GDP per capita is the most important factor, as well as total population and energy structure. With the corporate effect of Energy consumption per unit of GDP, the carbon intensity of energy of sub-sectors, sub-sector energy structure, GDP per capita and the total population, the increasing trend of $\mathrm{CO} 2$ emission of agricultural products processing industry has slowed down gradually.

According to the conclusions above, in order to reduce the carbon emission of agricultural product processing industry, measures can be taken in several aspects as follows:

i) Govern the industries by classification and enlarge the coverage of low carbon products for agricultural products processing industry. For the top three industries, specific governance should be taken to improve the whole industry's consciousness of low carbon.

ii) Improve the energy structure which mainly relies on coal at present. To change this situation, more new energy, such as gas, solar energy, wind energy, hydropower, and geothermal energy should be more used to reduce the emission of carbon and reach the goal of protecting the ecological environment.

iii) Agricultural products processing industries should focus more on technology research and development.

iv) Improve the energy efficiency of agriculture product processing industries.

Energy conservation, emission reduction and improving energy efficiency, as a key procedure in the process of our country's economic and social sustainable development, should be taken as a long-term strategy [3].

The energy consumption and carbon emission will be effectively reduced by these means, the short-term and long-term goals of agricultural products processing enterprises will also get perfect fusion.

\section{REFERENCES}

[1] Wang,Can,Chen,Jining,Zou,Ji,Decomposition of Energy-elated CO2 Emission in China:1957-2000.Energy, 30(1),pp.73-84,2005.

[2] Shi Dan, China's economic structure, growth pace and energy efficiency.

[3] International Pet roleum Economics, 15(7),pp. 7-15,2007.

[4] Xu Guo-quan,Liu Ze-yuan,Jiang Zhao-hua,Decomposition Model and Empirical Study of Carbon Emissions for China:19952004.China Population Resources And Environment, 16(6),pp. 158-161,2006.

[5] Xu Guang-yue,Song De-yong,An Empirical Research on the Relationship of Export Trade,Economic Growth and Carbon Emissions.Journal of International Trade,pp.74-79,2010.

[6] Shang Kai,Carbon Tax Policy Survey of Improving Carbondioxide Abatement in China.Shan Dong:Shan Dong University,2009.

[7] Aug BW,Zhang FQ,Choi KH,Factorizing Changes in Energy and Environmental Indicators through Decomposition.Energy, 23(6),pp.489-495,1998.

[8] Tang Jian-rong,Zhang Bai-yu,Wang Yu-hong,Research on Driving Factors of Carbon Emissions in China Based on LMDI.Statistics and Information Forum, 26(11),pp.19-26,2011. 